\title{
A LARGE APERTURE SUPERCONDUCTING DIPOLE FOR BETA BEAMS TO MINIMIZE HEAT DEPOSITION IN THE COIL
}

\author{
E. Wildner, C. Vollinger
}

\begin{abstract}
The aim of beta beams in a decay ring is to produce highly energetic pure electron neutrino and anti-neutrino beams coming from b-decay of ${ }^{18} \mathrm{Ne}^{10+}$ and ${ }^{6} \mathrm{He}^{2+}$ ion beams. The decay products, having different magnetic rigidities than the main ion beam, are deviated inside the dipole. The aperture and the length of the magnet have to be optimized to avoid that the decay products hit the coil. The decay products are intercepted by absorber blocks inside the beam pipe between the dipoles to protect the following dipole. A first design of a $6 \mathrm{~T}$ arc dipole using a cosine theta layout of the coil with an aperture of $80 \mathrm{~mm}$ fulfils the optics requirements. Heat deposition in the coil has been calculated using different absorber materials to find a solution to efficiently protect the coil. Aspects of impedance minimization for the case of having the absorbers inside the beam pipe have also been addressed.
\end{abstract}

CERN, Accelerator and Technology Department, Geneva, Switzerland

Presented at the 22nd Particle Accelerator Conference (PAC'07)

25-29 June 2007, Albuquerque, USA 


\title{
A LARGE APERTURE SUPERCONDUCTING DIPOLE FOR BETA BEAMS TO MINIMIZE HEAT DEPOSITION IN THE COIL
}

\author{
E. Wildner, CERN, Geneva, Switzerland \\ C. Vollinger, CERN, Geneva, Switzerland.
}

\section{Abstract}

The aim of beta beams in a decay ring is to produce highly energetic pure electron neutrino and anti-neutrino beams coming from b-decay of ${ }^{18} \mathrm{Ne}^{10+}$ and ${ }^{6} \mathrm{He}^{2+}$ ion beams. The decay products, having different magnetic rigidities than the main ion beam, are deviated inside the dipole. The aperture and the length of the magnet have to be optimized to avoid that the decay products hit the coil. The decay products are intercepted by absorber blocks inside the beam pipe between the dipoles to protect the following dipole. A first design of a $6 \mathrm{~T}$ arc dipole using a cosine theta layout of the coil with an aperture of $80 \mathrm{~mm}$ fulfils the optics requirements. Heat deposition in the coil has been calculated using different absorber materials to find a solution to efficiently protect the coil. Aspects of impedance minimization for the case of having the absorbers inside the beam pipe have also been addressed.

\section{INTRODUCTION}

To optimize the ratio between the straight sections and the arcs of a decay ring, proposed for the EURISOL project for neutrino production [1], a superconducting 6T dipole has been designed. The aim of such a decay ring is to produce highly energetic pure electron neutrino and anti-neutrino beams coming from $\beta$-decay of ${ }^{18} \mathrm{Ne}^{10+}$ and ${ }^{6} \mathrm{He}^{2+}$ ion beams. The design of the decay ring in the EURISOL proposal is based on the existing CERN infrastructure. The radioactive ion beams are extracted from the SPS as illustrated in Fig. 1.

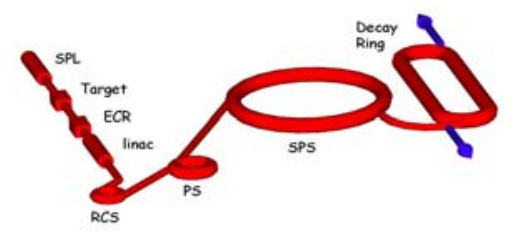

Figure 1: The decay ring with the injector chain.

The decay ring is a race track shaped storage ring, with a circumference of about $6900 \mathrm{~m}$, the same as the CERN SPS. The about $2500 \mathrm{~m}$ long straight sections, where the useful decay takes place, are oriented towards the experiments in the Frejus tunnel (France).

This preliminary feasibility study is limited to a "cosine theta" design, where as much as possible of existing ideas and technology has been used. The aperture of this dipole is large enough to house the ion beam and the decay products simultaneously. The dipole is protected against the decayed ions by absorbers inside the beam-pipe.

\section{REQUIREMENT FOR THE ARC DIPOLE}

The lattice in the two arcs of the decay ring is designed to limit the aperture needed for the dipoles; the optical function $\beta_{\mathrm{x}}$ and the dispersion have to be small. There are ten $38.7 \mathrm{~m}$ long cells in each arc. At the position where the decay products would hit the lattice dipole, following different trajectories due to the different magnetic rigidity, it is split into two independent dipoles. An absorber is inserted to intercept the decayed beams after each dipole. This way, the half-cell is composed of two dipoles and one quadrupole. The total arc length is $994 \mathrm{~m}$.

In Fig. 2 we show the two species decaying at the entrance of a dipole: the dipole length and aperture have been chosen to avoid that the beams impinge inside the dipole [1]. An absorber is placed where the aperture limit is reached. $\mathrm{Li}$ ions will be mostly abosorbed by the first dipole and the $\mathrm{F}$ by the second.

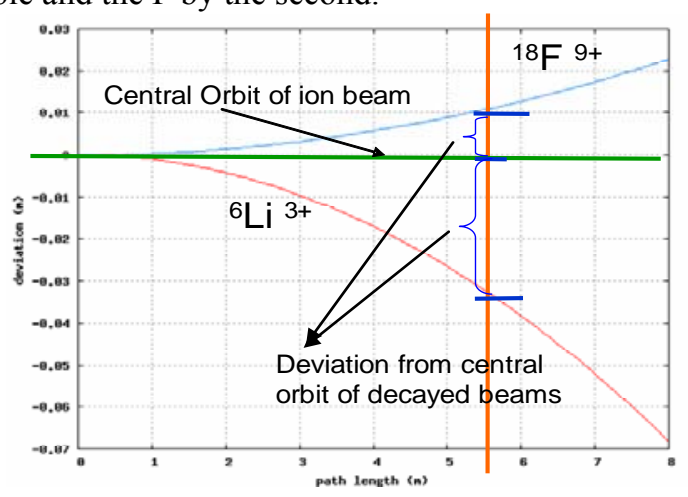

Figure 2: Decay products (child beams) and the ion beam (main beam) follow different trajectories due to different magnetic rigidity.

The choice is a dipole length of $5.7 \mathrm{~m}$ for a field of $6 \mathrm{~T}$. The main ion beam needs a half-aperture of $40 \mathrm{~mm}$, however the dipole aperture has to be large enough to avoid decays to impinge the vacuum chamber before it is intercepted by an absorber. These decaying ions are intercepted after the dipoles by an absorber. The decay products of ${ }^{18} \mathrm{Ne}^{10+}$ have to be intercepted after the second dipole in the half-cell.

Table 1: Requirements: Optics Parameters

\begin{tabular}{|l|l|l|}
\hline Parameter & Value & Unit \\
\hline Magn. rigidity of ion beam & 1000 & {$[\mathrm{Tm}]$} \\
\hline Bending radius & 156 & {$[\mathrm{~m}]$} \\
\hline Bending angle & $\mathrm{Pi} / 86$ & {$[\mathrm{rad}]$} \\
\hline Arc Dispersion & $10.98 /-0.24$ & {$[\mathrm{~m}]$} \\
\hline
\end{tabular}




\section{DESIGN OF THE MAIN BENDING DIPOLE}

\section{The Field Quality}

The main bending dipole magnet is a classical $\cos -\theta$ design with 5 blocks in two layers and a circular aperture of $80 \mathrm{~mm}$ radius. The $\mathrm{Nb}$-Ti cable which is used for the LHC main dipole inner layer has been chosen as a starting point due to its well-known characteristics that allowed us to omit a new cable design for this application. The layout of the magnet cross-section with the dipole field distribution is shown in Fig. 3. A simple circular iron yoke with an inner radius of $132 \mathrm{~mm}$ is surrounding the coil (not shown). The cable [2] is made of 28 superconducting strands and has an inner and outer width of 1.736 and $2.064 \mathrm{~mm}$, respectively which gives a slight keystoning. This keystoning, however, is not sufficient for a circular alignment around the mandrel which means that wedges are needed in the cross-section to achieve the required field quality.

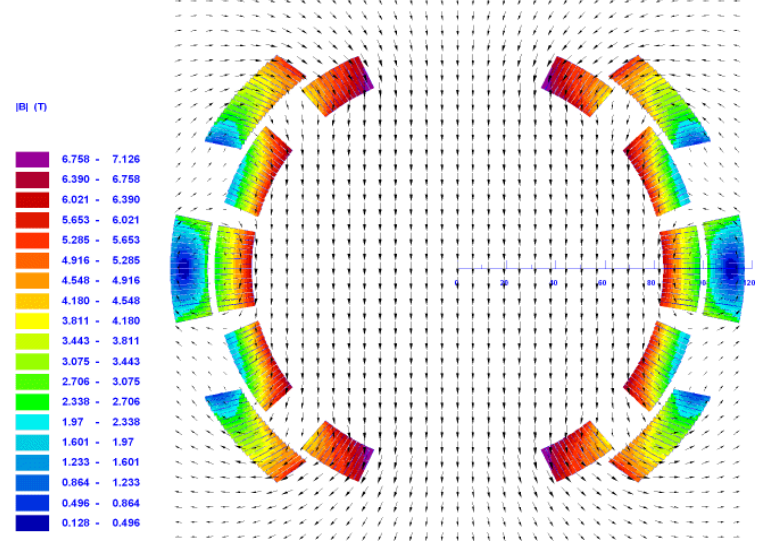

Figure 3: Cross-section of the main bending dipole with an $80-\mathrm{mm}$ aperture with main field lines and peak field distribution in the coil.

Space has been left for separating the two blocks of the outer layer which allows the insertion of a cooling pipe close to the inner layer, if needed. The required cooling will be calculated in a next step.

The multipoles, expressed in units of $10^{-4}$, are calculated from the magnetic field in the aperture as

$B_{\mathrm{y}}(x, y)+\mathrm{i} B_{\mathrm{x}}(x, y)=\sum_{n=1}^{\infty}\left(B_{\mathrm{n}}+\mathrm{i} A_{\mathrm{n}}\right)\left(\frac{x+\mathrm{i} y}{R_{\text {ref }}}\right)^{n-1}$,

where $\mathrm{x}$ and $\mathrm{y}$ are the transverse coordinates and $\mathrm{B}_{\mathrm{n}}$ and $A_{n}$ are the normal and skew multipoles calculated at a reference radius $R_{\text {ref }}$ relative to the main field $b_{n}=B_{n} / B_{1}$, $a_{n}=A_{n} / B_{1}$. From the simulation, we have reached a main field of $6.1 \mathrm{~T}$ with field errors at a reference radius of $53 \mathrm{~mm}$ (i.e., 2/3 of the aperture radius) given in Table 2 . Due to the coil symmetry in the simulation, the skew multipoles vanish.

The cable height is $15.1 \mathrm{~mm}$ and its critical current at $9 \mathrm{~T}, 1.9 \mathrm{~K}$ exceeds $13750 \mathrm{~A}$, giving a sufficiently large margin on the load line for this application that would allow even a small quantity of heat load to be taken by the coil without causing a quench, if needed. For block 3 (block with highest peak field of 7.1T in the crosssection), the margin on the load line is $73.2 \%$. The goal, however, is to intercept all particles by the absorbers after each dipole.

Table 2: Dipole Parameters

\begin{tabular}{|l|l|l|}
\hline Parameter & Value & Unit \\
\hline Central dipole field (nominal) & 6 & {$[\mathrm{~T}]$} \\
\hline Magnetic length & 5.7 & {$[\mathrm{~m}]$} \\
\hline Peak field in coil cross-section & 7.1 & {$[\mathrm{~T}]$} \\
\hline
\end{tabular}

The coil end has been designed in dimensions as compact as possible in order to leave sufficient space needed for the absorbers with a maximum peak field in the coil end of $6.3 \mathrm{~T}$.

\section{Forces in the Dipole Cross Section}

We carried out an estimation of the force distribution in the cross-section of the arc dipole to determine the stress on the coil midplane by calculating the azimuthal force in each conductor. The stress on the midplane adds up to $69 \mathrm{MPa}$ and $50 \mathrm{MPa}$ per quadrant in the inner and outer layer, respectively. The numbers correspond to a horizontal total force of $2.06 \mathrm{MN} / \mathrm{m}$ for both layers and a vertical total force of $0.84 \mathrm{MN} / \mathrm{m}$ which seems still feasible. (For comparison, in the LHC the coil is subjected to a horizontal force component per quadrant at nominal field of $1.8 \mathrm{MN} / \mathrm{m}$ and a vertical force component per quadrant at nominal field of $0.81 \mathrm{MN} / \mathrm{m}[2])$.

\section{HEAT DEPOSITION IN THE COIL}

For the stored beams in the decay ring, the lost power is around $10 \mathrm{~W} / \mathrm{m}$. Absorbers of non magnetic material have been inserted inside the beam-pipe, after each dipole, to intercept the peak losses loss, see Fig. 4.

The optics has been designed such that the major part of the decay products impinges on the absorbers. In the straight sections, the decay products are not bent by the dipole field and they follow the ion beam. When entering the dipole, all decayed ions accumulated in the straight sections form a beam of child particles following a different trajectory than the main ion beam. The child beams will be intercepted by the absorbers. It can be understood from Fig. 4 that in the second half of the second dipole, some of the beam decaying in the first dipole will not be absorbed. The absorbers between the magnets absorb the peaks of the decay products. The resulting heat deposition in the coils has been estimated.

The peak losses have been simulated using FLUKA $[5,6]$ by including in the model all particles decaying in the straight section up to the next dipole (1.4 $10^{9}$ nucleons/s). Care has been taken to check that, for this simple model, heat from the simulated beam is not deposited in the second half of the dipole, where we know from tracking simulations [1] that up to $10 \mathrm{~W} / \mathrm{m}$ energy is deposited from continuous beam loss in the dipole not taken into account in this simulation. This simulation only 
takes into account what impinges on the absorber from the accumulated beam spot, to calculate the absorption efficiency. The peak deposition from the $10 \mathrm{~W} / \mathrm{m}$ built up in the dipole has to be calculated separately.

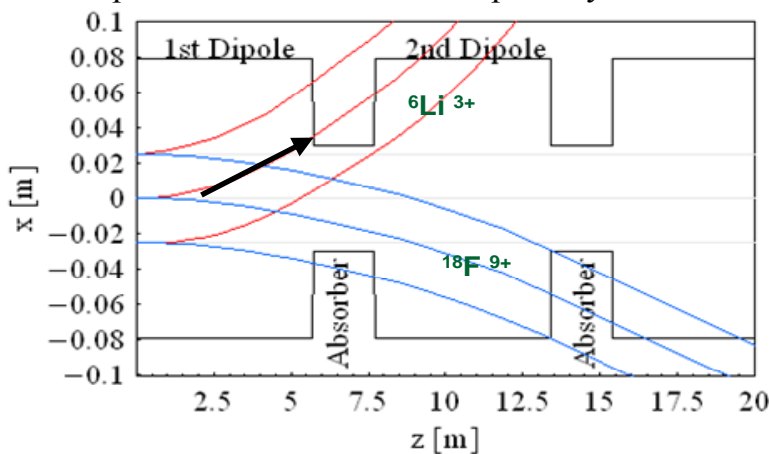

Figure 4: Top view of the beam chamber of two dipoles including the absorber. At the entrance of the first dipole the accumulated decay products are deviated from the central trajectory. The absorbers and the dipoles are designed to avoid these particles to hit the dipole. The black arrow on the first absorber represents the pencil beam used in the simulation. The absorber protects half the dipole according to tracking simulations [1].

A pencil beam $\left(1.410^{9}\right.$ nucleons/s) of ${ }^{6} \mathrm{Li}^{3+}$, accumulated in the arc straight section with the same momentum, $94 \mathrm{GeV} /$ nucleon, as the ion beam enters the dipole and impinges on the first absorber (Fig. 4). Further refined studies have to take into account all decayed particles along the machine by detailed tracking including the effect of the quadrupole field. In the model the coils are represented as a hollow cylinders inside the yoke, also cylindrical. The absorbers are $1 \mathrm{~m}$ long rings in the beam pipe. The beam-pipe and the insulation between the pipe and the coil are implemented.

We have checked the heat deposition using carbon, stainless steel and tungsten and also checked the case without absorber. Heat deposition has been scored in the dipole coil in cells with sizes corresponding to the transverse dimension of each cable $(15 \mathrm{~mm}$ radial and $1.5 \mathrm{~mm}$ azimuthal width and with a length of $20 \mathrm{~mm}$ ). This corresponds to local heat deposition in steady state operation. The results are shown in table 3, where the values in the cell with the highest heat deposition in the magnet coil are shown (Max Heat).

Table 3: Local heat deposition in the coil

\begin{tabular}{|c|c|c|c|}
\hline Material/Result & $\begin{array}{c}\text { Max Heat } \\
{[\mathrm{mW} / \mathrm{cm} 3]}\end{array}$ & $\begin{array}{c}\text { Distance } \\
{[\mathrm{cm}]}\end{array}$ & $\begin{array}{c}\text { Angle } \\
{[\text { degree] }}\end{array}$ \\
\hline Vacuum & $>30$ & $\sim 200$ & $\sim 0$ \\
\hline Carbon & 1.4 & 20 & 7 \\
\hline Stainless Steel & $<0.5$ & - & - \\
\hline Tungsten & $<0.5$ & - & - \\
\hline
\end{tabular}

The distance from the dipole entrance and the angular position of the maximum with respect to the mid plane is also shown. The distance of the maximum deposition should be in the first half of the magnet to avoid superposition of the around $10 \mathrm{~W} / \mathrm{m}$ found using tracking [2]. Statistical variations in the calculations are estimated to less that $0.5 \mathrm{~mW}$ and for stainless steel and tungsten the values found were below $0.5 \mathrm{~mW}$.

For comparison, for the LHC magnet energy deposition limit related to the quench threshold $\left(12 \mathrm{~mW} / \mathrm{cm}^{3}\right)$ in the coil is $4.3 \mathrm{~mW} / \mathrm{cm}^{3}$, including necessary margins. The maximum is located in the magnet mid plane.

\section{FUTURE WORK AND CONCLUSION}

The proposed design [1] appears to be feasible to achieve with a superconducting dipole. The dipole design has been carried out in order to provide data for the energy deposition study and should be further optimized. The absorber is capable of intercepting the spot beam accumulated in the straight part before the dipole.

Cryogenic aspects of the dipole and the heat deposition still have to be addressed. The absorbers inside the beam pipe may be a problem for impedances seen by the beam, unless a liner is introduced, see Fig. 5. First estimates [3] give indications that this would be acceptable, however further studies are needed.

The dipole design should be optimized and the heat deposition calculated using a detailed model including a model of the quadrupole and using detailed tracking models [4] to define the parameters of the particles impinging the superconducting magnets.

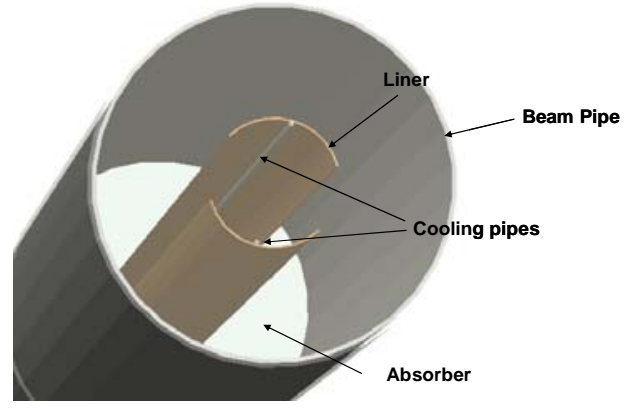

Figure 5: Beam pipe with liner inside, supported by the absorber.

\section{REFERENCES}

[1] A. Chancé, J. Payet, "First design for the optics of the decay ring for the beta-beams", April 2006, EURISOL report DS/TASK12/TN-06-05

[2] LHC Design Report, Vol. 1, page 157, ISBN 929083-224-0

[3] Erk Jensen, CERN, private communication

[4] F.W. Jones, TRIUMF, "Development of the ACCSIM tracking and simulation code", PAC 1997

[5] A Fasso, A Ferrari, J Ranft, and PR Sala, "FLUKA: a multi-particle transport code", CERN-2005-10 (2005), INFN/TC_05/11, SLAC-R-773

[6] A. Fasso, A. Ferrari, S. Roesler, P.R. Sala, G. Battistoni, F. Cerutti, E. Gadioli, M.V. Garzelli, F. Ballarini, A. Ottolenghi, A. Empl and J.Ranft, "The physics models of FLUKA: status and recent developments", Computing in High Energy and Nuclear Physics 2003 Conference (CHEP2003), La Jolla, CA, March, 2003 